\title{
Resistance and Seakeeping Investigation for Optimization of the Floating Hull of Wave Glider
}

\author{
Alaaeldeen M. E. Ahmed', Wenyang Duan² \\ ${ }^{1}$ Shipbuilding Engineering Department, Military Technical College, Cairo, Egypt \\ ${ }^{2}$ College of Shipbuilding Engineering, Harbin Engineering University, Harbin, China \\ Email: robyhadad@yahoo.com, Duanwy2004@126.com
}

How to cite this paper: Ahmed, A.M.E. and Duan, W.Y. (2016) Resistance and Seakeeping Investigation for Optimization of the Floating Hull of Wave Glider. World Journal of Engineering and Technology, 4, 235-249.

http://dx.doi.org/10.4236/wjet.2016.43D029

Received: September 30, 2016

Accepted: October 22, 2016

Published: October 29, 2016

\begin{abstract}
Wave glider is the first unmanned autonomous marine robot to use only the ocean's endless supply of wave energy for propulsion. Wave glider comprises fin system, tether and float which harvest all of its energy from waves and sun to produce forward thrust. As a consequence of the lack of design information and data for the wave glider, the main aim of the study is using computational fluid dynamics (CFD) to present a method to predict calm water resistance for the floating hull through calculations of 3 different hull forms using the same mesh generation under the same conditions. Calculations are carried out using 3 different mesh sizes for Froude number in the range of 0.10 to 0.40 and compared for accuracy of the solution parameters. Wigley parabolic hull, high speed round bilge form (NPL) and Series 60 have been comparatively investigated in order to estimate the hydrodynamics performance of the hull. The linear seakeeping analysis, coupled heave and pitch motions, roll motion, in irregular waves, with one parameter Bretschneider and JONSWAP spectra. Numerical computations have been performed for motion response predictions of the three hulls which cover wave angles from $0^{\circ}$ to $180^{\circ}$ at $45^{\circ}$ intervals for six different forward speeds from 0 to 4.304 knots using Maxsurf Motion software. The close agreement between the numerical predictions shows the importance of $C F D$ applications in estimating the hydrodynamics performance to design the floating hull and the numerical method is useful in glider design. The fine grid is fit to the calculation and shows the most appropriate results because convergent results are obtained as the mesh size decrease so the fine grid is the one which will be applied for the other hulls. Also it can be observed that the added resistance and the RAOs for NPL hull are less than the other hulls. Therefore from the comparisons, the NPL hull is the optimum hull compared to the other hulls from the resistance and seakeeping point of view.
\end{abstract}




\section{Keywords}

Wave Glider, Calm Water Resistance, Seakeeping, CFD, Maxsurf

\section{Introduction}

The wave glider demonstrates a new Unmanned Surface Vehicles (USV) platform with unique capabilities for continuous data collection, in a complex marine environment and could operate with no costly deep water mooring or shipping operations. Wave glider is a hybrid sea surface and underwater vehicle consisting of a submerged glider, attached via a tether, to a surface float. The vehicle is propelled by the submerged glider which can convert the vertical ocean wave motion energy into forward thrust as shown in Figure 1. All other electrical devices are powered by solar panels attached to the float. As this vehicle was designed specifically to collect data from the ocean, the vehicle is capable of accommodating various kinds of sensors which may be used for different applications during missions [1]. The vehicle transmits data to land in real-time sequence, allowing for accurate and easy monitoring of the vehicle's trip and the data collected [2].

$C F D$ technology is always developing, especially in terms of new solution techniques and these developments are given in terms applicable to the ship building industry [3]. The main aim of the study is to investigate the CFD capability in solving fluid flow around the floating part of the wave glider. The method is validated on three standard ship hull forms which are wigley hull, NPL and series $60(2 \mathrm{~m})$. Together with the free surface shape, the calm water resistance is determined and the calculated results were compared by using commercial general purpose software fluent which solves the Reynolds-averaged Navier-stokes (RANS) equations with a finite-volume approach on hybrid structured grids in an attempt to design the floating hull [4] [5].

Then; to further predict the seakeeping trends for hull forms as well as increase the accuracy of information, a continuation of this study looking at the three hulls should be conducted. Doing this would produce a solid base of information on how a large variety of accepted hull forms are expected to perform as a floating hull in the seas [6]. Another tool to increase the analysis is Maxsurf Motions software which could vary the wave amplitude, change the ship's velocity or wave direction as well as look at other modal period ranges [7]. The objective of this paper is to bring accurate numerical
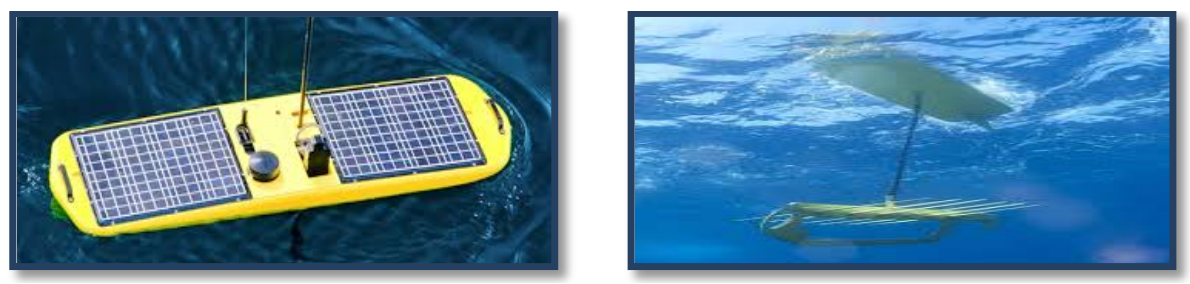

Figure 1. wave glider showing the surface vehicle and submerged glider (www.bing.com/images/waveglider). 
prediction of hull resistance and seakeeping performance closer to early stage design using a method that is flexible and applicable to high-performance hull form types.

\section{Mathematical Model and Governing Equations}

The first critical step is to select an appropriate hull forms upon which a realistic wave glider hull model may be configured. The idea behind using wigley hull, NPL and series 60 models is the availability of the numerical and experimental data and their simple geometrical form. They are also mathematically defined and have a history in being used for validation [8] [9]. CFD results of wigley hull model are calculated and compared to NPL and series 60 using the same mesh generation method under the same conditions to ensure proficiency in $C F D$ theory [10].

Volume of fluid (VOF) formulation and the open channel boundary conditions available in fluent are applied to solve multiphase free-surface flow. To solve the governing equations for fluid flow, the fluid domain is subdivided into a finite number of cells and these equations are changed into algebraic form via discretion process. The coupling between the pressure and velocity fields was achieved using simple (semi-implicit methods for pressure-linked equation) algorithm. The shear-stress transport (SST) $\mathrm{k}-\omega$ turbulence model is adopted to calculate eddy viscosity in our study; this model is believed to be one of the best choices to simulate turbulence flow around ship hull [11].

In this study, three-dimensional unsteady, incompressible, two-phase (air and water) and viscous turbulent flow field is investigated. For incompressible flow, well known, continuity and Navier-Stokes equations over a control volume are given by:

$$
\begin{gathered}
\frac{\partial U i}{\partial x i}=0 \\
\frac{\partial(\rho U i U j)}{\partial x j}=-\frac{\partial p}{\partial x i}+\frac{\partial}{\partial x j}\left[\mu\left(\frac{\partial U i}{\partial x j}+\frac{\partial U j}{\partial x i}\right)-\left(\frac{2}{3} \mu \frac{\partial u j}{\partial x i}\right)\right]-\frac{\partial}{\partial x j}\left(\rho u_{i}{ }^{\prime} u_{j}{ }^{\prime}\right) .
\end{gathered}
$$

where $u_{i}$ and $u_{j}$ are time-averaged velocity components $(i, j=1,2,3), \rho$ is fluid density, $\mathrm{p}$ is pressure and $\mu$ is dynamic viscosity. In this equation, $-\rho \mathrm{u}_{\mathrm{i}}{ }^{\prime} \mathrm{u}_{\mathrm{j}}{ }^{\prime}$ is called Reynolds stress which must be modeled in order to close the equations. A turbulence model is required here in order to close the system of RANS equations. SST k- $\omega$ model is developed by Menter [12]. Transport Equations for the SST k- $\omega$ Model are:

$$
\begin{gathered}
\frac{\partial}{\partial t}(\rho \mathrm{k})+\frac{\partial}{\partial \mathrm{x}_{\mathrm{i}}}\left(\rho \mathrm{ku}_{\mathrm{i}}\right)=\frac{\partial}{\partial \mathrm{x}_{\mathrm{j}}}\left(\Gamma_{\mathrm{k}} \frac{\partial \mathrm{k}}{\partial \mathrm{x}_{\mathrm{j}}}\right)+\mathrm{G}_{\mathrm{k}}-\mathrm{Y}_{\mathrm{k}}+\mathrm{S}_{\mathrm{k}} \\
\frac{\partial}{\partial \mathrm{t}}(\rho \omega)+\frac{\partial}{\partial \mathrm{x}_{\mathrm{i}}}\left(\rho \omega \mathrm{u}_{\mathrm{i}}\right)=\frac{\partial}{\partial \mathrm{x}_{\mathrm{j}}}\left(\Gamma_{\omega} \frac{\partial \omega}{\partial \mathrm{x}_{\mathrm{j}}}\right)+\mathrm{G}_{\omega}-\mathrm{Y}_{\omega}+\mathrm{D}_{\omega}+\mathrm{S}_{\omega}
\end{gathered}
$$

In these equations, $G_{k}$ represents the generation of turbulence kinetic energy due to mean velocity gradients, $G_{\omega}$ represents the generation of $\omega, \Gamma_{k}$ and $\Gamma_{\omega}$ represent the effective diffusivity of $k$ and $\omega$ respectively. $Y_{k}$ and $Y_{\omega}$ represent the dissipation of $k$ and $\omega$ due to turbulence. $D_{\omega}$ represents the cross-diffusion term, $S_{k}$ and $S_{\omega}$ are user-defined source terms. 
Maxsurf Motions uses linear strip theory method to predict the vessels heave and pitch response. Roll response is estimated assuming that the vessel behaves as a simple, damped, spring/mass system, and that the added inertia and damping are constant with frequency. The first step in the assessment of seakeeping performance is usually to determine the wave spectrum for a seaway [13]. Wave spectrum is the spectral representation of wave elevation which based on time is converted so that it can be represented as function of frequency by using FFT integration technique.

The wave assumed to be long crested that incident on the vessel. The way in which the energy of the sea distributed at various encounter frequencies is given by the wave spectrum $S_{\zeta}\left(\omega_{e}\right)$. By the principle of linear superposition, the sea spectrum can be related to the motion spectrum through the response amplitude operator (RAO) which is then computed for each critical mode of motion. The RAO defines the amplitude of response due to unit wave excitation. If the transfer function at various encounter frequencies are designated RAO, the response spectra is $S_{r}\left(\omega_{e}\right)$ then the response spectra of the vessel in that particular seaway is given by:

$$
\mathrm{S}_{\mathrm{r}}\left(\omega_{\mathrm{e}}\right)=\mathrm{S}_{\zeta}\left(\omega_{\mathrm{e}}\right) \times|\mathrm{RAO}|^{2}
$$

\section{Computational Domain and Grid Generation}

The three hull models used for this study are standard for ship-hydrodynamics research and they are used by ITTC research programs. The characteristics and the longitudinal profile of the hull of each mode used for $C F D$ calculations are tabulated in Table 1.

Water density for hulls; is $101.930 \mathrm{~kg} \cdot \mathrm{sec}^{2} / \mathrm{m}^{4}$ and kinematic viscosity is $1.2845 \times 10^{-6}$ $\mathrm{m}^{2} / \mathrm{sec}$.

A complete $C F D$ solution requires three major steps: preprocessing, analysis of the problem using a solver and post processing of the results [14]. In this study, preprocessing of the solution involves geometry creation of the model and an appropriate mesh generation is carried out in gambit software. The geometry is obtained by the use of offset tables and the structured hexahedral grid of each hull has been produced for the numerical investigations as shown in Figures 2-4 for wigley hull, NPL and series 60 respectively [15] [16]. These offsets data are imported into gambit in the form of vertices and then 3-D models are generated. Unstructured tetrahedral was not chosen since this type of mesh reduces accuracy and increases the computational cost despite of the fact that it is easy to handle while dealing with complex geometry [17].

Table 1. Characteristics of wigley parabolic hull, NPL and Series 60.

\begin{tabular}{ccccc}
\hline Description & Units & Wigley hull & NPL & Series 60 \\
\hline Water plane length & $\mathrm{L}_{\mathrm{w}}(\mathrm{m})$ & 2.00 & 2.00 & 2.00 \\
Maximum water plane breadth & $\mathrm{B}_{\mathrm{w}}(\mathrm{m})$ & 0.30 & 0.28 & 0.28 \\
Maximum depth & $\mathrm{D}_{\mathrm{M}}(\mathrm{m})$ & 0.30 & 0.30 & 0.30 \\
Maximum draft & $\mathrm{d}_{\mathrm{M}}(\mathrm{m})$ & 0.12 & 0.11 & 0.11 \\
Wetted surface area & $\mathrm{S}\left(\mathrm{m}^{2}\right)$ & 0.69 & 0.54 & 0.73 \\
Volume of displacement & $\nabla\left(\mathrm{m}^{3}\right)$ & 0.03 & 0.03 & 0.035 \\
Block coefficient & $\mathrm{C}_{\mathrm{B}}$ & 0.373 & 0.406 & 0.658 \\
\hline
\end{tabular}



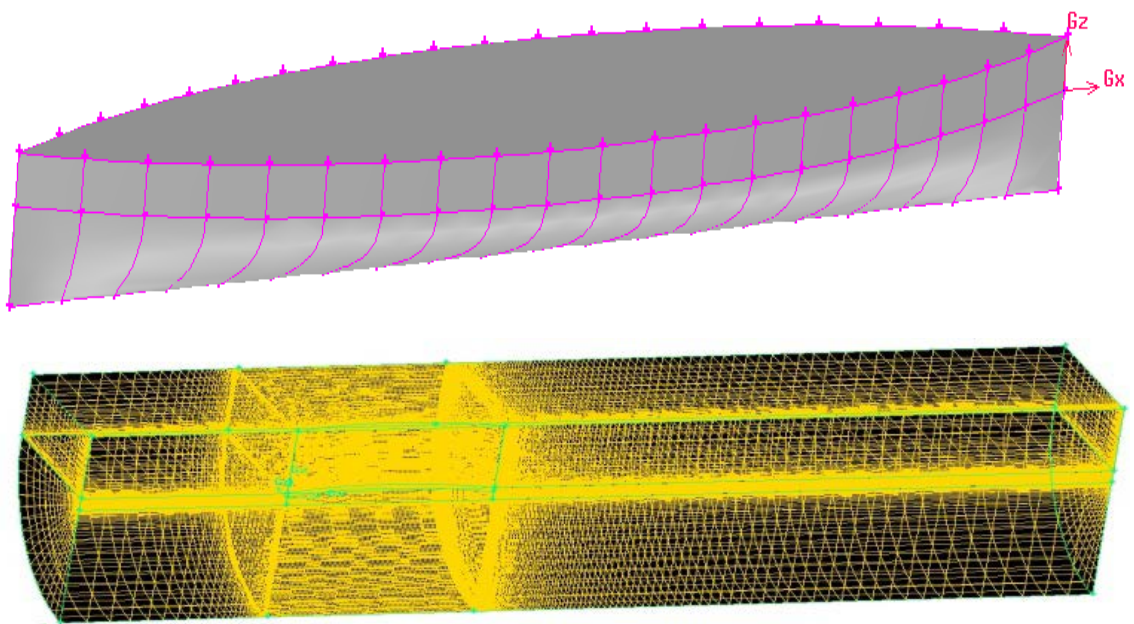

Figure 2. Geometry and the meshed domain volume in gambit for wigley hull.
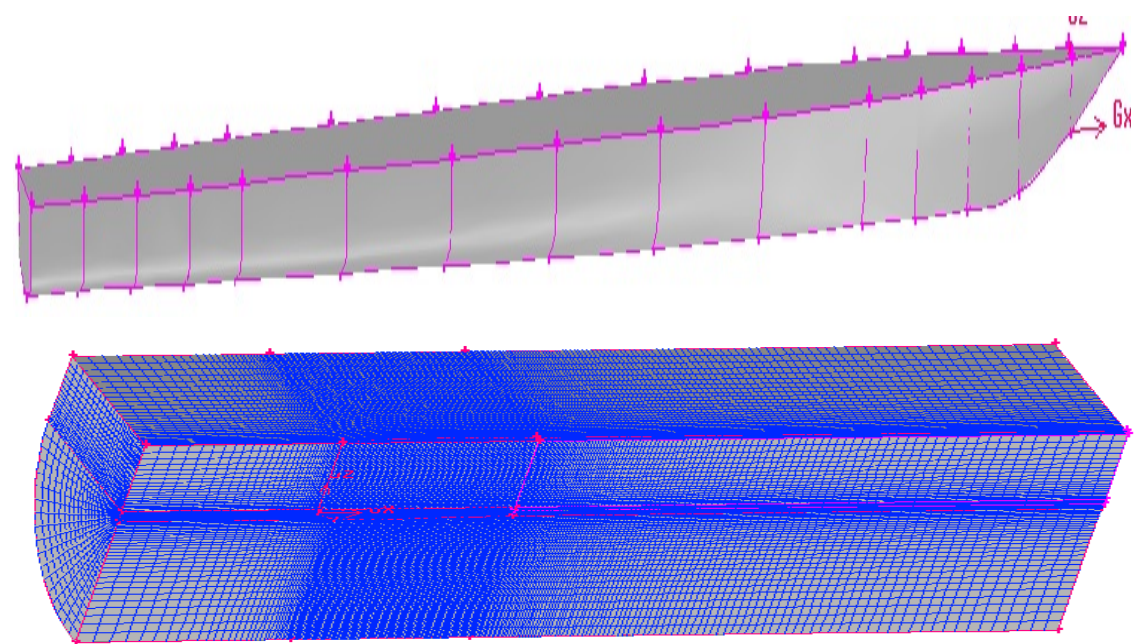

Figure 3. Geometry and the meshed domain volume in gambit for NPL.
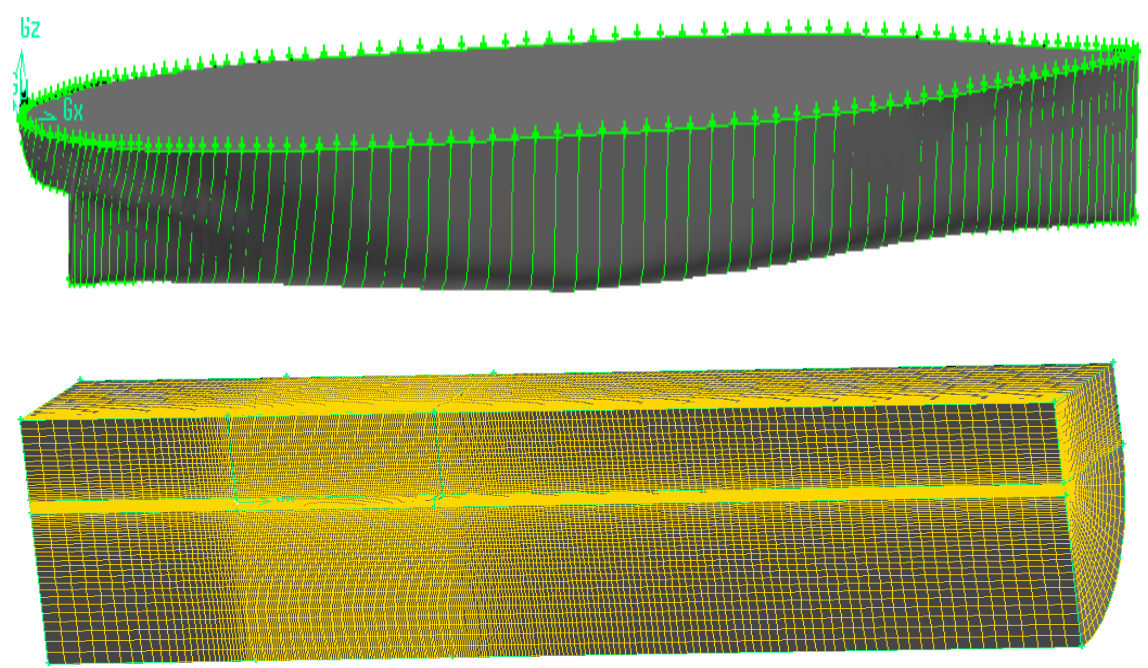

Figure 4. Geometry and the meshed domain volume in gambit for Series 60 . 
For flow calculations, half of the three hulls are modeled due to the symmetry of hull. The domain volume is taken to be of cylinder shape. The overall size of the cylinder is 5 $\mathrm{L} \times 1.5 \mathrm{~L} \times \mathrm{L}$. The Cartesian coordinate system is setup with $\mathrm{x}$-axis pointing towards the bow, $y$-axis to portside, and $\mathrm{z}$-axis upwards. The length in front of the hull (inlet boundary) and behind the hull (outlet boundary) are taken as $1.0 \mathrm{~L}$ and $3.0 \mathrm{~L}$ (main hull) respectively. The top is taken $0.5 \mathrm{~L}$, bottom and side boundaries are taken as $1.0 \mathrm{~L}$ (main hull) each, the domain volume is subdivided into various sub volumes in order to obtain structured multi-block grid.

Three different mesh sizes are evaluated in this study; the total elements are 275,220 for coarse grid, 400,620 for medium grid and 427,940 for fine grid for wigley hull, 293,220 for coarse grid, 437,820 for medium grid and 445,620 for fine grid for NPL form, 285,400 for coarse grid, 416,920 for medium grid and 441,280 for fine grid for NPL form.

A seakeeping analysis of three floating hulls $2.00 \mathrm{~m}$ length are performed and carried on with an original Maxsurf software program based on linear seakeeping method and statistical short term prediction response method. For flow calculations, the domain volume is taken to be of cubical shape. The coordinate system is setup with $\mathrm{x}$-axis pointing towards the forward, $y$-axis to starboard and $z$-axis upwards. The number of sections, buttocks and waterlines for each hull are 81 sections, 21 buttocks and 11 lines respectively as shown in Figures 5-7.
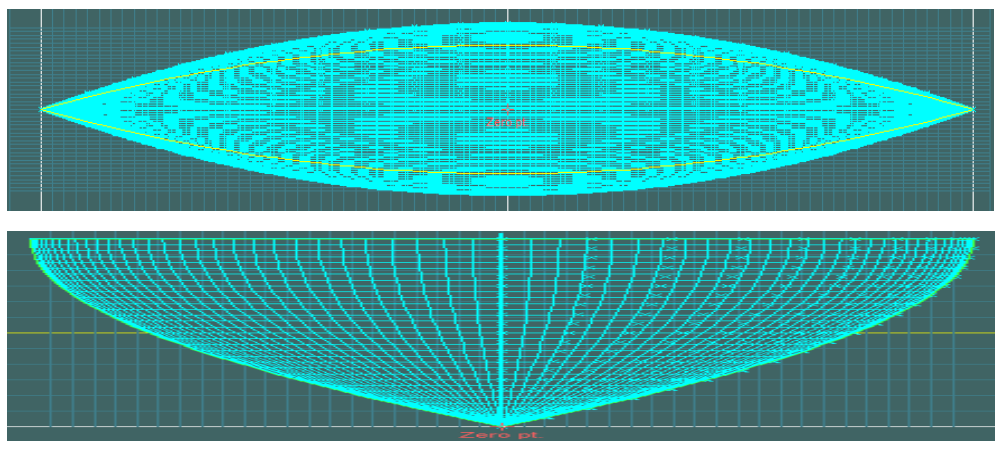

Figure 5. The plan view and the body plan of wigley hull using Maxsurf.
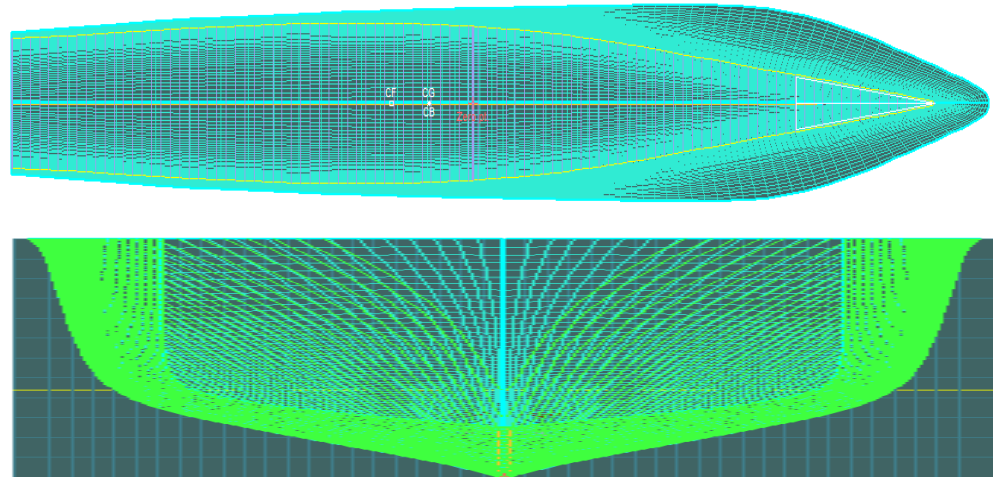

Figure 6. The plan view and the body plan of NPL using Maxsurf. 

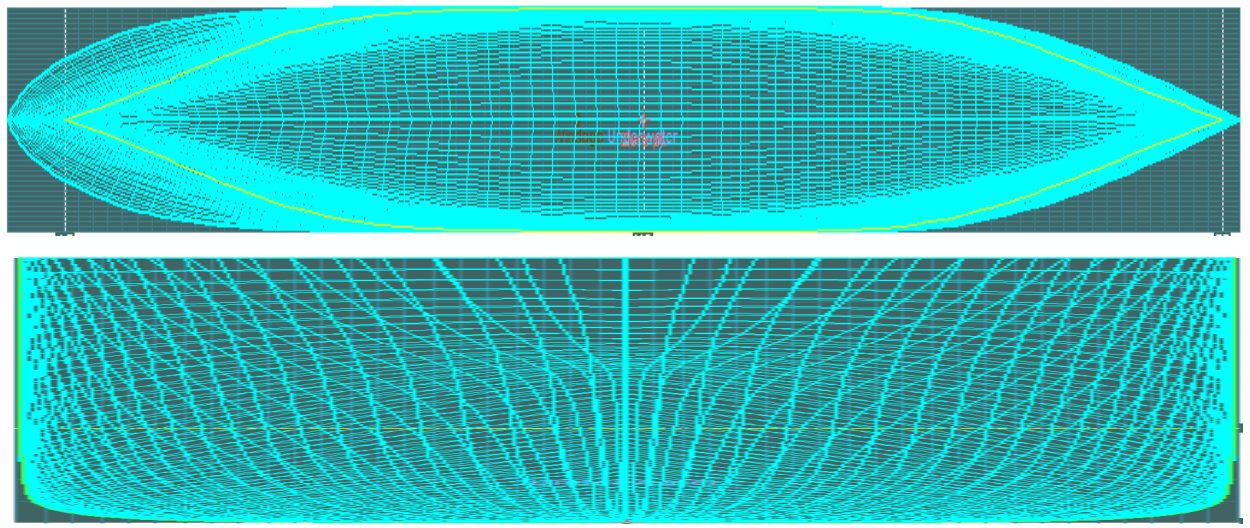

Figure 7. The plan view and the body plan of Series 60 using Maxsurf.

\section{Boundary Conditions and Solution Parameters}

The fluent software is used for computations in this study. Since the motion of the freesurface is governed by gravitational and inertial forces, therefore, gravity effects must be taken into account in boundary conditions. The turbulence models of SST k- $\omega$ with standard coefficients are employed in the calculations. The inlet boundary condition upstream and the outlet boundary condition downstream are taken as pressure-inlet and pressure-outlet with open channel while the flow velocity is considered equal to the experimental velocity of the model. No-slip wall boundary condition is taken on the whole surfaces. Symmetry condition is invoked on the symmetric plane.

Convergence of the solution is assessed by monitoring the residuals of continuity, velocity, turbulence, volume fraction and drag force. The residual convergence criterion is taken as $1 \mathrm{e}^{-07}[18]$.

\section{Resistance Calculations}

In this study; a comparison between the numerical predictions of the three hull forms is done to evaluate a mesh generation method to design the floating hull of wave glider. The calm water resistance of the wigley hull, NPL and series $60(2 \mathrm{~m})$ were calculated versus Froude number ranging from 0.10 to 0.40 under the same conditions by using $C F D$ as shown in Figure 8 and Figure 9.

For mesh study, the difference between total resistances for the three hulls is generally acceptable. The grid results are in very close agreement. The difference of the predicted resistance is very small and of the same order of magnitude. So the mesh generation method is applicable and the close agreement between the numerical predictions shows that the overall numerical scheme is suitable for resistance prediction.

From the grid results, the fine grid is fit to the calculation and shows the most appropriate results because convergent results are obtained as the mesh size decrease so the fine grid is the one which will be applied for the other hulls. Wave glider is designed with slender hull shapes in order to decrease the wave making resistance of the ship. It can be observed from the comparisons that the NPL hull is the optimum hull, not only because it has simple geometrical form and also mathematically defined, simple and has 

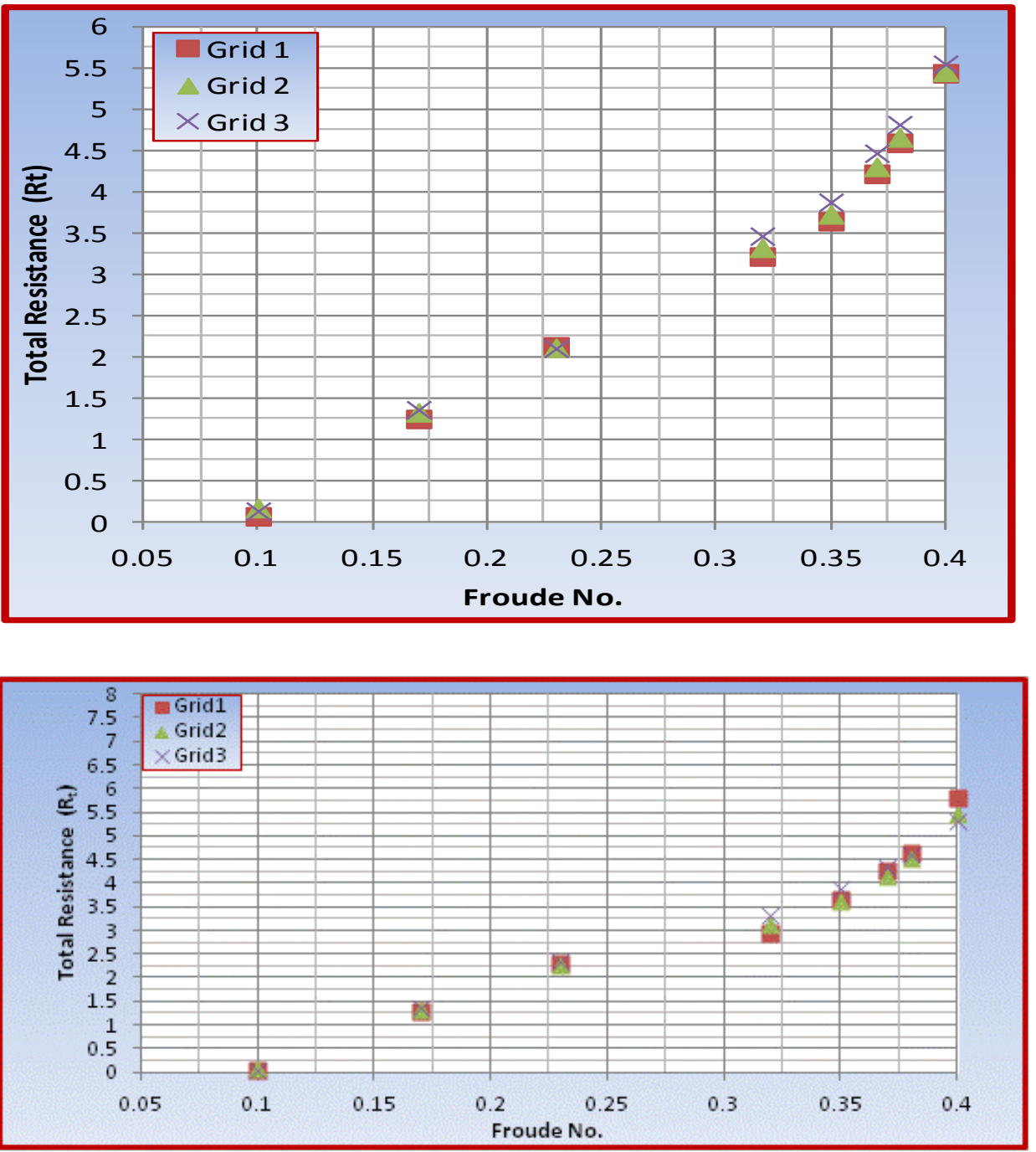

Figure 8. Resistance curve for wigley hull and series 60 comparing three different sized grids respectively.

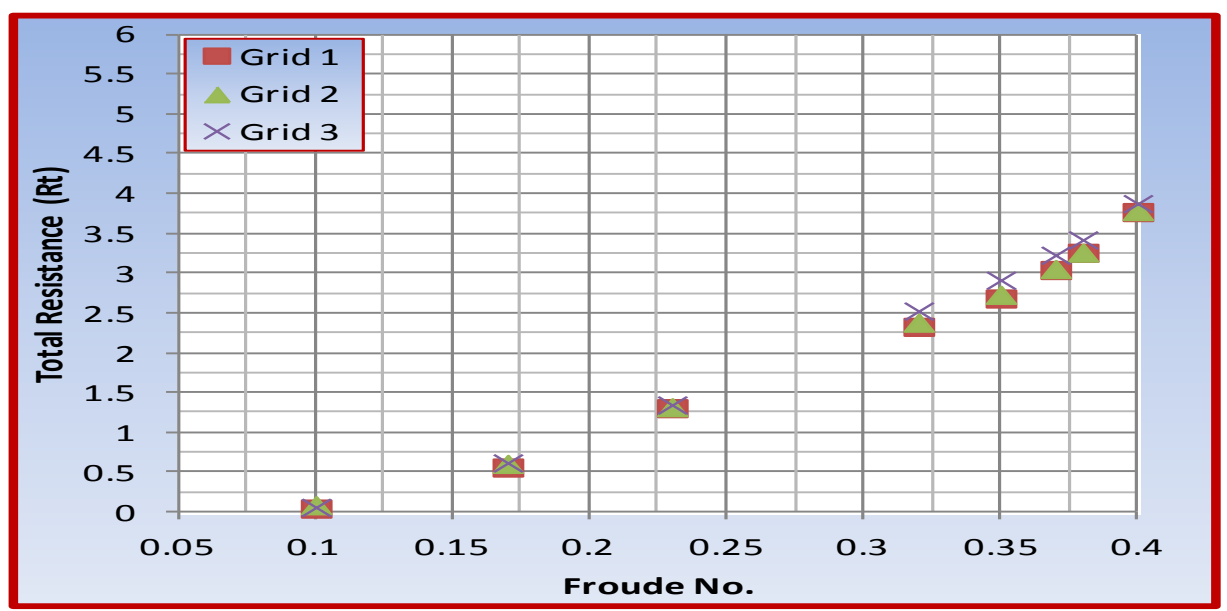

Figure 9. Resistance curve for NPL comparing three different sized grids respectively. 
a long history in being used for validation but also has low resistance as compared to the other hulls. The calculated drag on the hull was recorded and plotted in order to judge the convergence of the solution. The coarse, medium and fine grid results are in very close agreement [19].

Finally Contours for free surface wave and volume fraction (water) for the four hull models model at various Froude number are shown in Figures 10-12 respectively.

\section{Seakeeping Calculations}

The study includes the linear seakeeping analysis, coupled heave and pitch motions, roll motion in irregular waves, heading angles varied from $0^{\circ}$ to $180^{\circ}$, with one parameter Bretschneider and JONSWAP wave power density spectrums which always have a peak enhancement factor of 1.0 and 3.30 respectively. Taking into account the specific limits of seakeeping criteria, the dynamic response statistical polar diagrams are obtained for each motion degree and the cumulative one, pointing out the influence of the shipspeed and heading angle for seakeeping assessment. The two parameters that varied between programs runs were the wave angle and ship speed. Each run only changed one of the two parameters at a time. The ship's speed was varied from Fn $=0$ to Fn $=0.5$ in increments of Fn $=0.1$ (approximately from 0 to 4.304 knots in increments of 0.861 knots). Due to space limitations, only the two primary responses in heave and pitch are presented. A hull that possesses favorable response characteristics in both heave and pitch, will in general exhibit acceptable seakeeping behavior in all other responses.
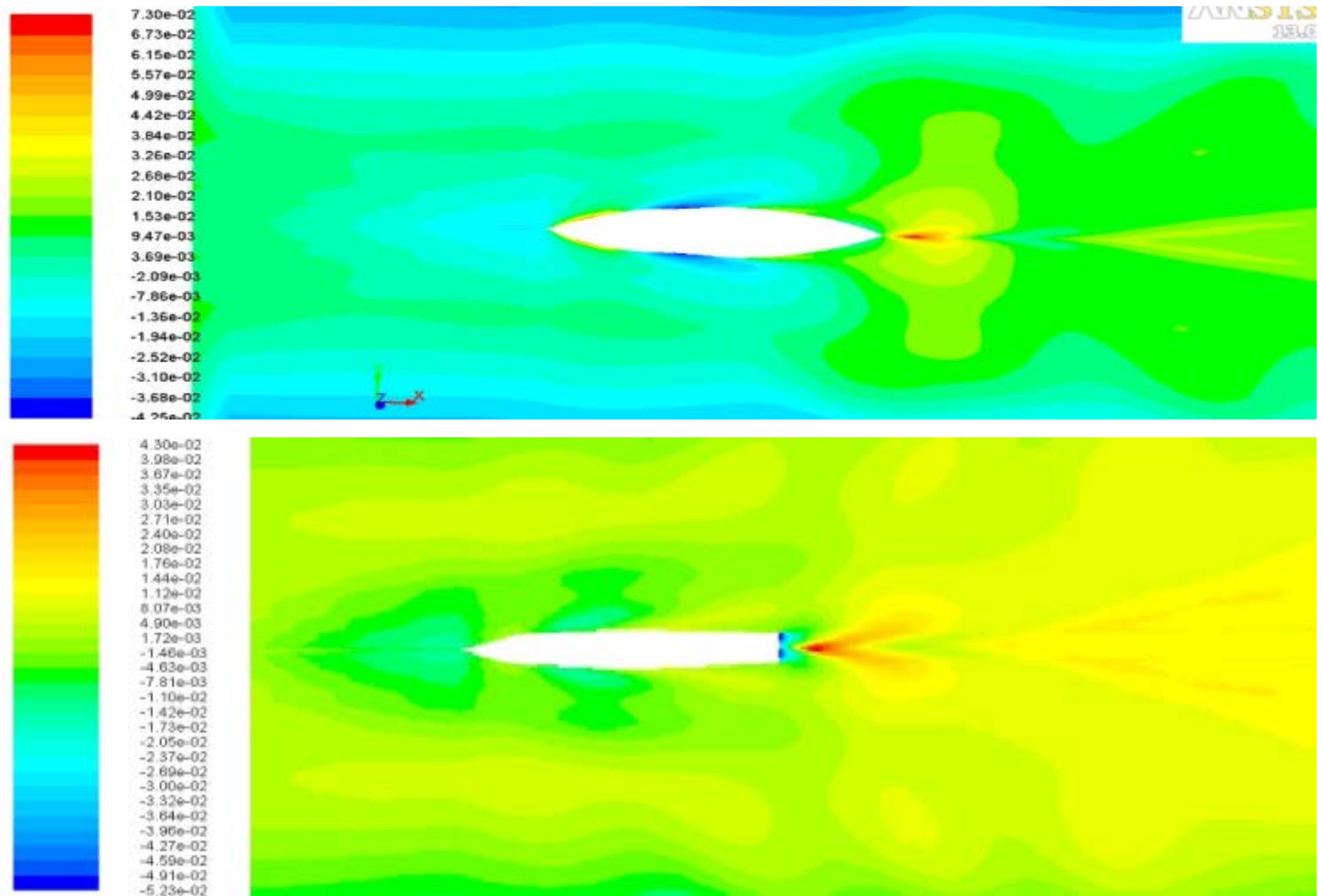

Figure 10. Free surface wave contours for wigley hull and NPL at Fn $=0.40$ coarse grid respectively. 

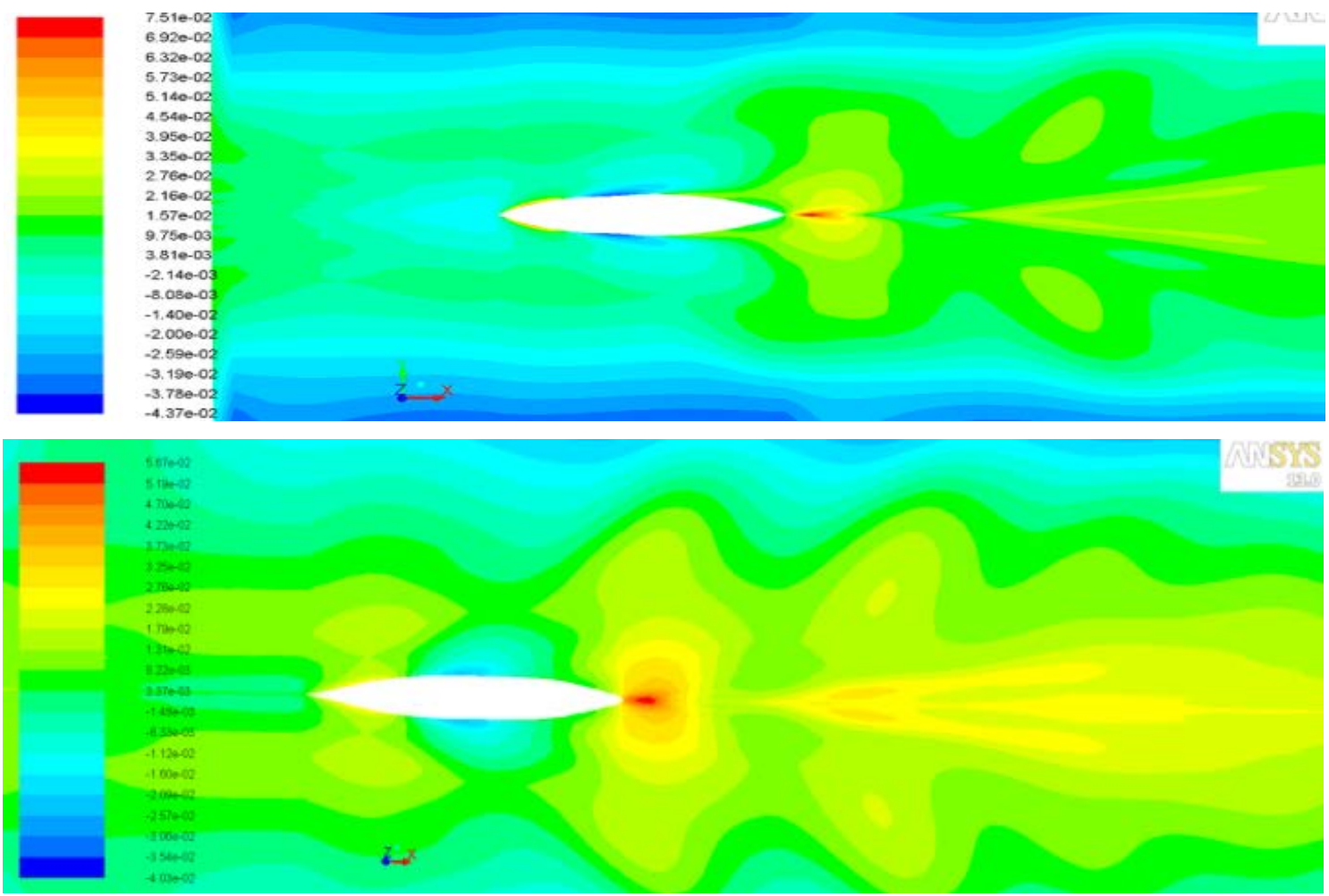

Figure 11. Free surface wave contours for wigley hull and series 60 at $\mathrm{Fn}=0.40$ medium grid respectively.

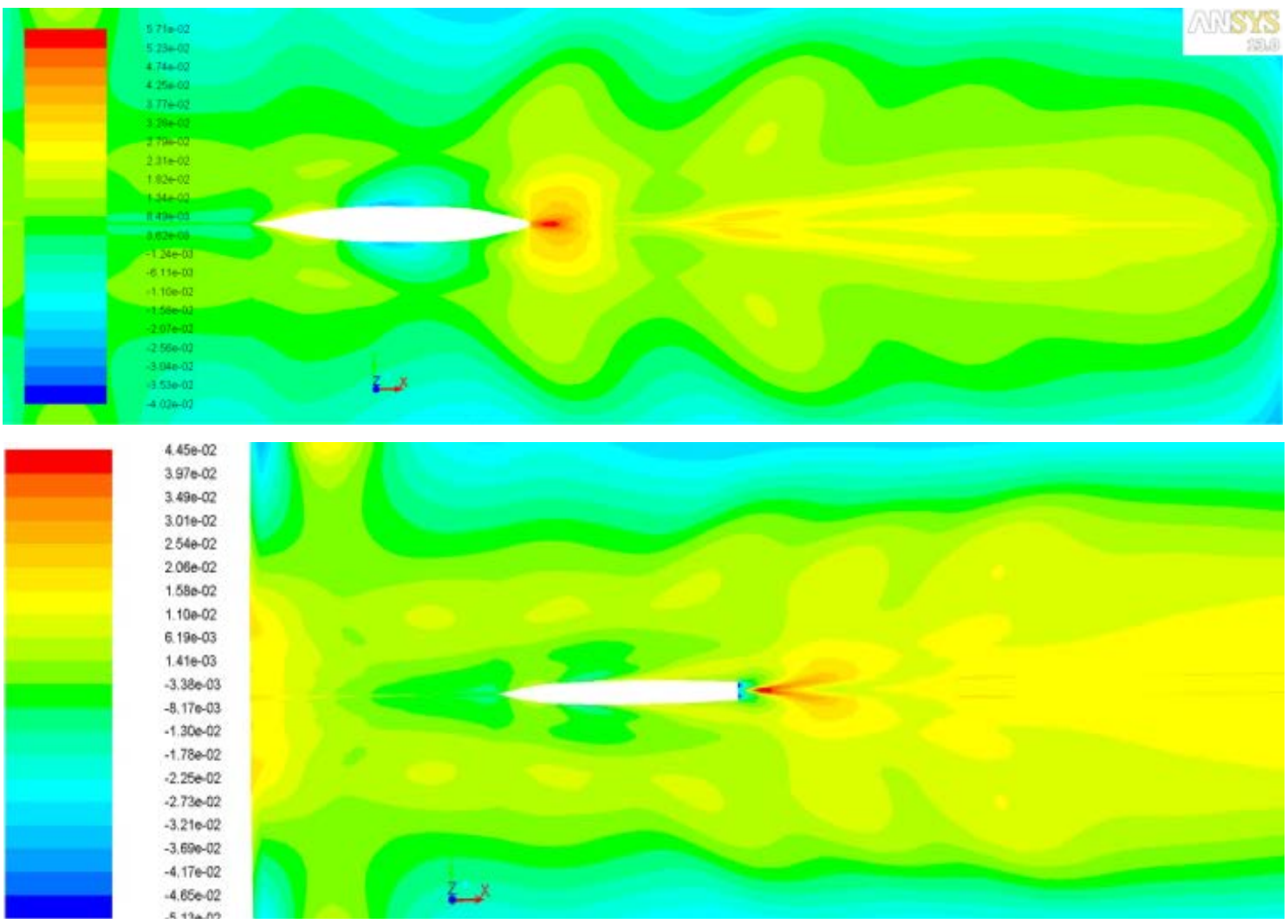

Figure 12. Free surface wave contours for series 60 and NPL at Fn $=0.40$ fine grid respectively. 
The variation of motion characteristics with increasing sea state can be established and added resistance for the floating hull is calculated. The objective of this study is to analyze the ship speed and heading angle influence on maximum RMS heave, pitch, and roll motion and acceleration amplitudes [20].

The range of vessel headings analysis was varied from $0^{\circ}$ to $180^{\circ}$ using increment vessel headings of $45^{\circ}$, this produced satisfactory results and the solving time was not excessive. It should be noted that assuming vessel symmetry about the longitudinal centre line is necessary to analyze between $0^{\circ}$ and $180^{\circ}$ of vessel headings.

Figure 13 shows the speed polar plots for the NPL form in smooth sea state using the one param. Bretschneider spectrum. The plots for the three hulls show that the wave response patterns from the models are the same but with varying levels of intensity where a suitable scale illustrates the intensity of the motion. The plots also illustrate the hull's motion based on the direction of the waves interacting with the hull which is oriented at 180 degrees and the speed of the ship which increases from the center to the outer ring. Figure 13 also shows that the heave response is higher in beam and head seas while the roll motion is highest in the beam sea. The pitch response is highest in stern and head seas. From the results, it is noted that roll has a peaky response spectra in the region near to its natural frequency. Whereas, for pitch the response is more flattened and dispersed. The RAOs for heave and roll are quite consistent with their maximum responses occurring near their natural frequencies. However, for pitch, as expected RAOs obtained were not consistent. This is as a consequence of the flattened and disperses nature of response spectrum for pitch.

Numerical results and the measurements of the floating hull agree quite well in frequency and time domain. This paper provides important information regarding the seakeeping of the wave glider hull and how to be used in numerous scenarios. The results indicate that the choice of seakeeping performance depends on the nature of seakeeping analysis and criteria-free measures offer a simple, practical, and realistic assessment in comparative seakeeping studies. Finally, the main analysis of the results obtained is in the form of spectral analysis of wave and hull motions. From them the RAO can be obtained, which is the key to all seakeeping analysis.

Figures 14-16 show the wave surface contour diagrams for different headings $0^{\circ}$, $45^{\circ}, 90^{\circ}, 135^{\circ}$ and $180^{\circ}$ for wigley parabolic hull, NPL and series 60 hull for both cases regular and irregular wave surface respectively.

\section{Conclusion}

This paper demonstrates investigation of the optimum hull used to design the floating hull of wave glider from the resistance and seakeeping point of view. After comparing CFD calculations results for total resistance between the three hull forms, we conclude that the NPL has the lowest resistance compared to the other hulls. Also from the grid results, the fine grid is fit to the calculation and shows the most appropriate results because convergent results are obtained as the mesh size decrease. So the fine grid can be used to design the floating part of the wave glider (the hull). Furthermore, the good 
RMS Pitch motion [deg]

$\square \mathrm{Fn}=0,0 \mathrm{kn}$

$\square F_{n}=0.1,0.881 \mathrm{kn}$

$F n=0.2,1.721 \mathrm{kn}$

$F n=0.3,2.582 \mathrm{kn}$

$F n=0.4,3.443 \mathrm{kn}$

$\mathrm{Fn}_{\mathrm{n}}=0.5,4.304 \mathrm{kn}$

\section{Added resistance [kN]} $\square \mathrm{Fn}_{\mathrm{n}}=0,0 \mathrm{kn}$

$\square F_{n}=0.1,0.881 \mathrm{kn}$

$F_{n}=0.2,1.721 \mathrm{kn}$

$F_{n}=0.3,2.582 \mathrm{kn}$

$F n=0.4,3.443 \mathrm{kn}$

$\mathrm{Fn}=0.5,4.304 \mathrm{kn}$
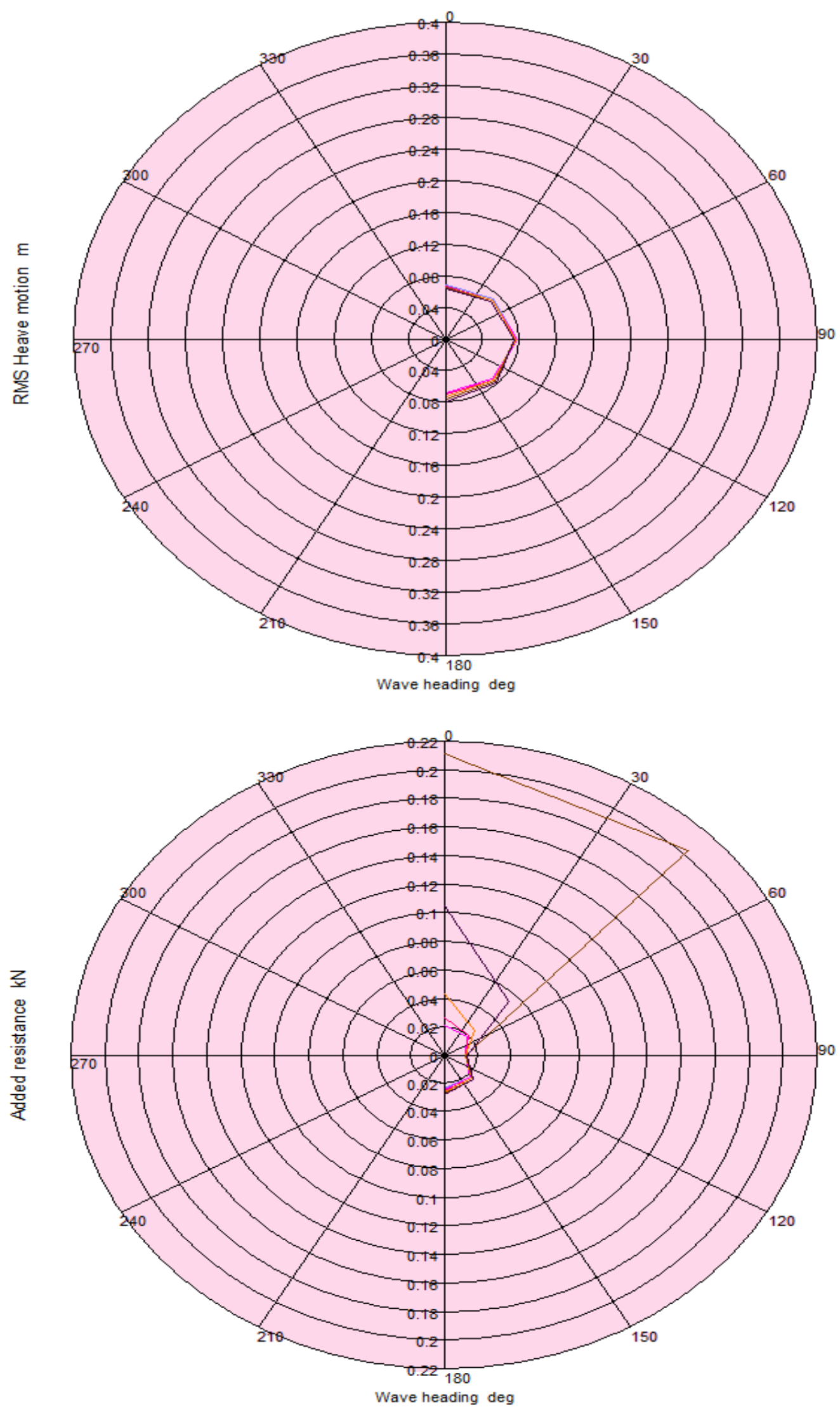


\begin{tabular}{|l} 
RMS Pitch motion [deg] \\
$F n=0,0 \mathrm{kn}$ \\
$F n=0.1,0.881 \mathrm{kn}$ \\
$F n=0.2,1.721 \mathrm{kn}$ \\
$F n=0.3,2.582 \mathrm{kn}$ \\
$F n=0.4,3.443 \mathrm{kn}$ \\
$F n=0.5,4.304 \mathrm{kn}$
\end{tabular}

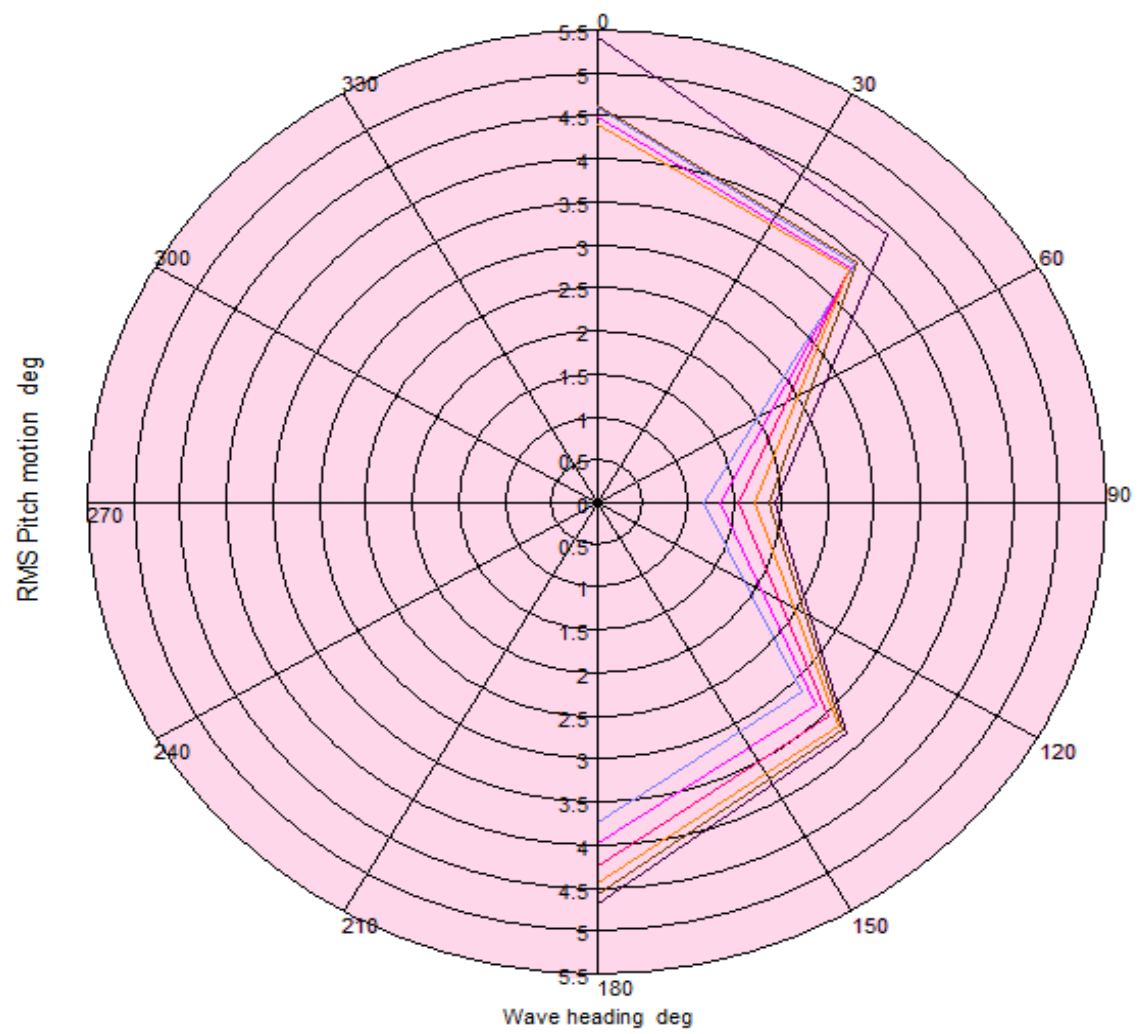

\begin{tabular}{|l} 
RMS Roll motion [deg] \\
$F n=0,0 \mathrm{kn}$ \\
$F n=0.1,0.881 \mathrm{kn}$ \\
$F n=0.2,1.721 \mathrm{kn}$ \\
$F n=0.3,2.582 \mathrm{kn}$ \\
$F n=0.4,3.443 \mathrm{kn}$ \\
$F n=0.5,4.304 \mathrm{kn}$
\end{tabular}

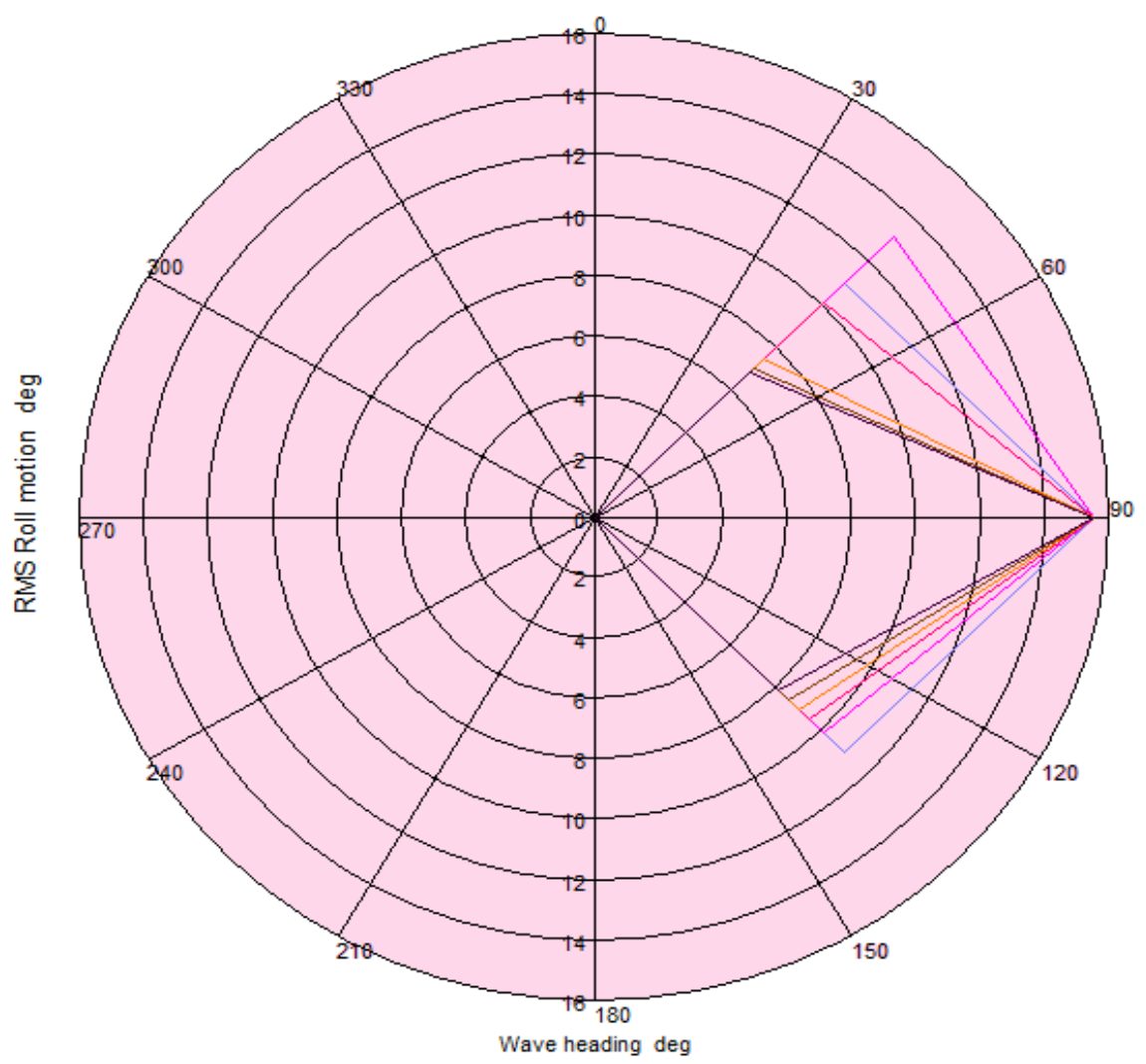

Figure 13. Contour plots for NPL hull at head seas, 180 deg; smooth (1 param. Bret.: $2.74 \mathrm{~s}, 0.3 \mathrm{~m})$. 

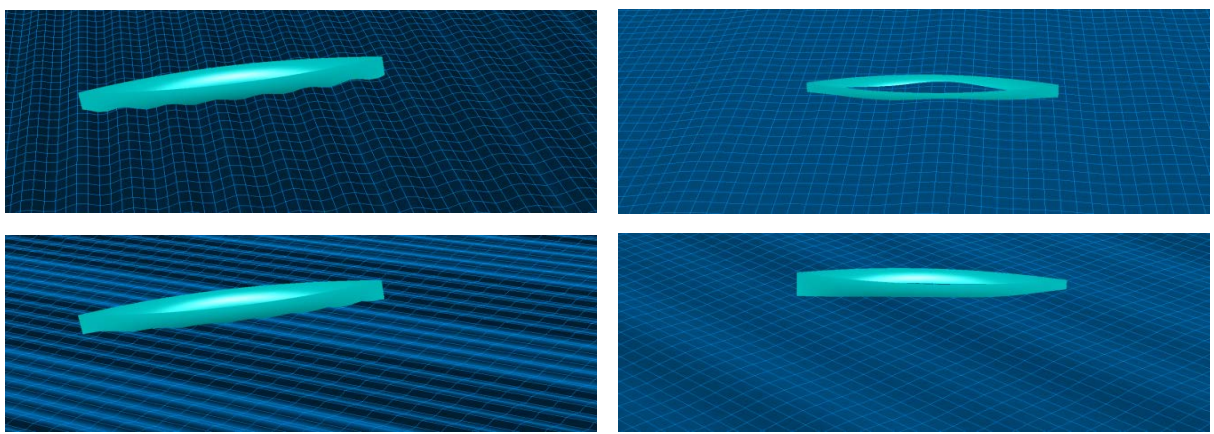

Figure 14. Wave surface contour diagrams for different headings $0^{\circ}$ and $45^{\circ}$ for wigley hull (column 1 regular wave surface), (column 2 irregular wave surface).
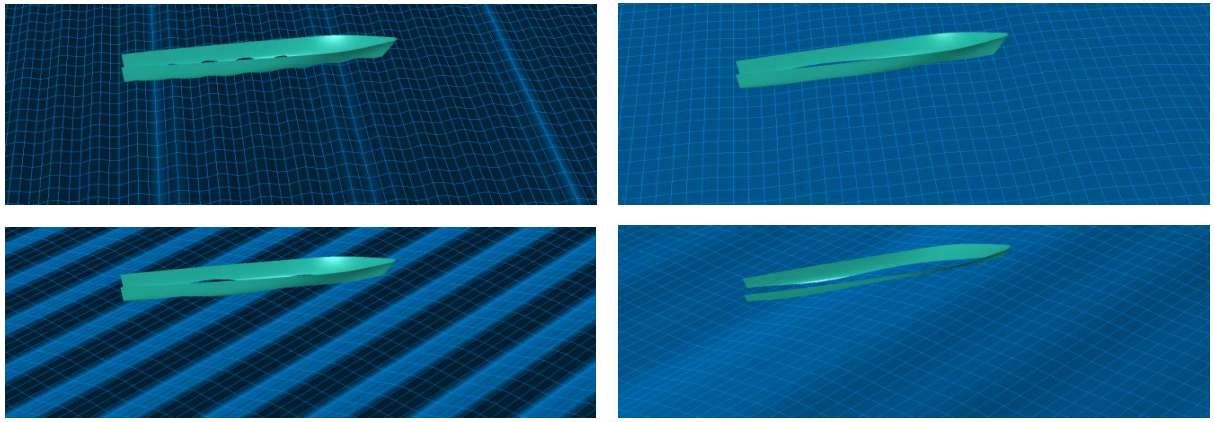

Figure 15. Wave surface contour diagrams for different headings $0^{\circ}$ and $135^{\circ}$ for NPL hull.
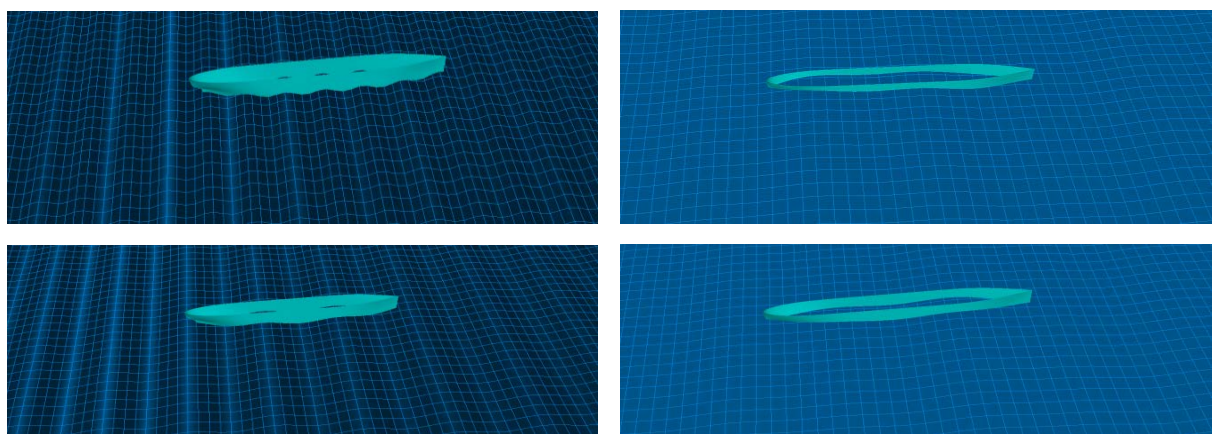

Figure 16. Wave surface contour diagrams for different headings $0^{\circ}$ and $180^{\circ}$ for series 60 .

agreement in results showed the importance of CFD applications in estimating the hydrodynamics performance to design the floating hull.

Based on the numerical results, we noted that the added resistance results of NPL hull is lower than the other hulls at different velocities under the same conditions. Also it may be seen that the RAOs for the NPL are slightly less than wigley hull and series 60 form. However, it is necessary to calculate the actual seakeeping characteristics in the expected sea conditions to ensure the results that NPL is the best floating hull for the wave glider.

\section{References}

[1] Hine, et al. (2012) U. S. Patent 8,287,323 B2. 
[2] Daniel, T., Manley, J. and Trenaman, N. (2011) The Wave Glider: Enabling a New Approach to Persistent Ocean Observation and Research. Ocean Dynamics, 61, 1509-1520. http://dx.doi.org/10.1007/s10236-011-0408-5

[3] Özdemir, Y.H., Bayraktar, S. and Yılmaz, T. (2007) Computational Investigation of a Hull. 2nd International Conference on Maritime Research and Transportation ICMRT.

[4] Ansys (2008) Fluent 13 User's Guide, Fluent Inc.

[5] Wigley, W. (1934) A Comparison of Experimental and Calculated Wave Profiles and Wave Resistances for a Form Having Parabolic Waterlines. Proc. Roy. Soc. London, Ser. A, 144, 144-159. http://dx.doi.org/10.1098/rspa.1934.0039

[6] Lloyd, A.R.J.M. (1989) Seakeeping: Ship Behavior in Rough Weather. Ellis Harwood Limited, Chichester.

[7] Pawlowski, J. (1991) A Theoretical and Numerical Model of Ship Motions in Heavy Seas. In SNAME Transactions, 99, 319-315.

[8] Calkins, D.E., Schachter, R.D. and Olivera, L.T. (2001) An Automated Computational Method for Planning Hull Form Definition in Concept Design. Ocean Engineering, 28, 297 327. http://dx.doi.org/10.1016/S0029-8018(99)00069-4

[9] Barrass, C.B. (2004) Ship Design and Performance for Masters and Mates. Butter-WorthHeinemann, 218-227.

[10] Ishikawajima-Harima Heavy Industries Co., Ltd. (1983) Technical Report, Cooperative Experiments on Wigley Parabolic Models in Japan. Ship Research Institute. University of Tokyo.

[11] Rosemurgy, W.J., Edmund, D.O., Maki, K.J. and Beck, R.F. (2011) A Method for Resistance Prediction in the Design Environment. 11th International Conference on Fast Sea Transportation, Honolulu, September.

[12] Menter, F.R. (1994) Two-Equation Eddy-Viscosity Turbulence Models for Engineering Applications. AIAA Journal, 32, 1598-1605. http://dx.doi.org/10.2514/3.12149

[13] (2013) Maxsurf Motions Program and User Manual, Bentley Systems.

[14] Peric, M. and Ferziger, J.H. (2002) Computational Methods for Fluid Dynamics. 3rd Edition, Springer.

[15] Marwood, W. and Bailey, D. (1969) Design Data for High-Speed Displacement Hulls of Round-Bilge Form. Ship Report 99, National Physical Laboratory.

[16] Bailey, D. (1976) The NPL High Speed Round Bilge Displacement Hull Series, Marine Technology Monograph, The Royal Institute of Naval Architects (RINA), Vol. 4.

[17] Lin, C.W., Percival, S. and Gotimer, E.H. (1995) Viscous Drag Calculations for Ship Hull Calculations. Research Report.

[18] Zhang, Z.R., Liu, H., Zhu, S.P. and Zhao, F. (2006) Application of CFD in Ship Engineering Design Practice and Ship Hydrodynamics. Journal of Hydrodynamics, Ser. B, 18, 315-322.

[19] Alaaeldeen, A., Duan, W. and Deng, R. (2014) A Computational Fluid Dynamics Method for Resistance Prediction of the Floating Hull of Wave Glider. Advanced Materials Research, 936, 2114-2119. http://dx.doi.org/10.4028/www.scientific.net/AMR.936.2114

[20] Sarioz, K. and Narli, E. (2004) Effect of Criteria on Seakeeping Performance Assessment. Elsevier Ireland Ltd. 
Submit or recommend next manuscript to SCIRP and we will provide best service for you:

Accepting pre-submission inquiries through Email, Facebook, LinkedIn, Twitter, etc. A wide selection of journals (inclusive of 9 subjects, more than 200 journals)

Providing 24-hour high-quality service

User-friendly online submission system

Fair and swift peer-review system

Efficient typesetting and proofreading procedure

Display of the result of downloads and visits, as well as the number of cited articles

Maximum dissemination of your research work

Submit your manuscript at: http://papersubmission.scirp.org/

Orcontactwjet@scirp.org 